\title{
Minor Stroke Syndromes in Large-Vessel Occlusions: Mechanical Thrombectomy or Thrombolysis Only?
}

\author{
(D) M.P. Messer, (DS. Schönenberger, (D) M.A. Möhlenbruch, (D). Pfaff, DC. Herweh, DP.A. Ringleb, and (D) S. Nagel
}

\begin{abstract}
SUMMARY: While mechanical thrombectomy for large-vessel occlusions is now an evidence-based treatment, its efficacy and safety in minor stroke syndromes (NIHSS $\leq 5$ ) is not proved. We identified, in our prospective data base, 378 patients with minor strokes in the anterior circulation; 54 (14.2\%) of these had proved large-vessel occlusions. Eight of 54 (14.8\%) were immediately treated with mechanical thrombectomy, 6/54 (11.1\%) after early neurologic deterioration, and the rest were treated with standard thrombolysis only. Rates of successful recanalization were similar between the 2 mechanical thrombectomy groups (75\% versus 100\%). Rates of excellent outcome (modified Rankin Scale 0-1) were higher in patients with immediate thrombectomy $(75 \%)$ compared with patients with delayed thrombectomy (33.3\%) and thrombolysis only (55\%). No symptomatic intracranial hemorrhage occurred in either group. These descriptive data are encouraging, and further analysis of large registries or even randomized controlled trials in this patient subgroup should be performed.
\end{abstract}

ABBREVIATIONS: IV = intravenous thrombolysis only; MSS = minor stroke syndrome; MT = mechanical thrombectomy; END = early neurologic deterioration; MT-I = mechanical thrombectomy immediately; sICH = symptomatic intracranial hemorrhage

$I^{\mathrm{n}}$ addition to thrombolysis, mechanical thrombectomy has become the standard of care for patients presenting with ischemic strokes due to large-vessel occlusions in the anterior circulation. ${ }^{1}$ In general, large-vessel occlusions are associated with severe stroke symptoms indicated by high National Institutes of Health Stroke Scale scores. However, large-vessel occlusions may also present as a minor stroke syndrome (MSS).

Three of the 5 seminal thrombectomy studies enrolled patients with a baseline NIHSS $\geq 6$ (Endovascular Treatment for Small Core and Proximal Occlusion Ischemic Stroke [ESCAPE], Endovascular Revascularization With Solitaire Device Versus Best Medical Therapy in Anterior Circulation Stroke Within 8 Hours [REVASCAT]) or $\geq 8$ (Solitaire With the Intention for Thrombectomy as Primary Endovascular Treatment [SWIFTPRIME]), therefore excluding MSS. ${ }^{1-4}$ The Multicenter Randomized Clinical Trial of Endovascular Treatment for Acute Ischemic Stroke in the Netherlands (MR CLEAN) and Extending the Time for Thrombolysis in Emergency Neurological Deficits-Intra-Ar-

Received November 2, 2016; accepted after revision January 31, 2017.

From the Departments of Neurology (M.P.M., S.S., P.A.R., S.N.) and Neuroradiology (M.A.M., J.P., C.H.), University of Heidelberg, Heidelberg, Germany.

Please address correspondence to Simon Nagel, MD, Department of Neurology, University Hospital Heidelberg, INF 400, 69120 Heidelberg, Germany; e-mail: simon.nagel@med.uni-heidelberg.de

三 Indicates article with supplemental on-line table.

http://dx.doi.org/10.3174/ajnr.A5164 terial (EXTEND-IA) trials did not set a lower NIHSS cutoff but included only a small number of patients with MSS, resulting in a mean baseline NIHSS of $17 .{ }^{1,5,6}$ Hence, it is unclear whether patients presenting with MSS due to large-vessel occlusions may benefit from thrombectomy. Here we present a retrospective and descriptive analysis of a case series derived from a large prospective stroke data base, with particular focus on mechanical thrombectomy performed immediately after presentation with MSS or as rescue therapy after clinical deterioration.

\section{Case Series}

We searched our prospective data base between 1998 and 2015 for patients with ischemic strokes due to large-vessel occlusions in the anterior circulation who received an acute recanalization therapy and presented with MSS. All patients were treated according to current in-house standard operating procedures in either our stroke unit or neurointensive care unit. Clinical baseline, radiologic data, and outcome data were obtained from the data base. Recanalization was assessed with the TICI scale by 2 blinded investigators on a consensus basis, and successful recanalization was defined as $\geq$ TICI $2 \mathrm{~b}$. The outcome was measured with the modified Rankin Scale after 3 months and was obtained through rehabilitation reports, outpatient assessments, or a standardized interview by an unblinded investigator. Symptomatic intracranial hemorrhage (sICH) was defined according to the European Cooperative Acute Stroke Study-II (blood at any site in the brain and 


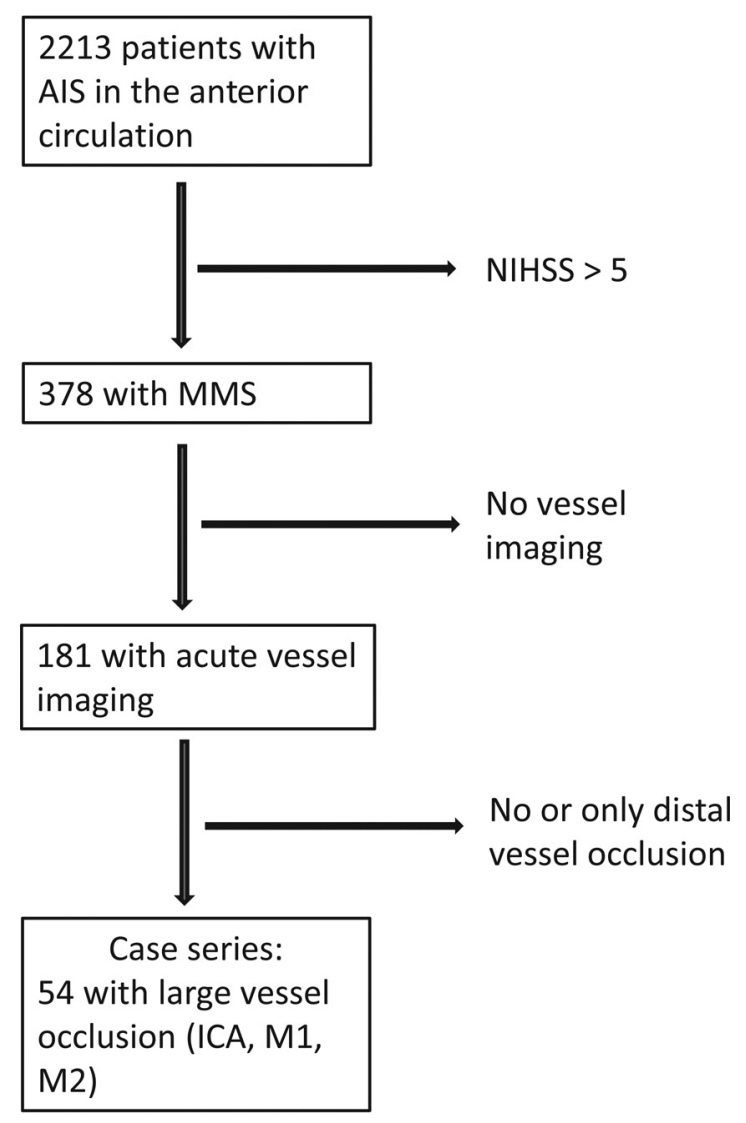

FIG 1. Flow chart of the patient inclusion criteria, indicating how patients were selected for the case series.

clinical deterioration with an increase in the NIHSS score of at least 4 points compared with the lowest value within the first 7 days or any intracranial hemorrhage leading to death). ${ }^{7}$ Early neurologic deterioration (END) was defined as an increase of the NIHSS of $\geq 4$ points within the first 24 hours. Patients with MSS with occlusions of the internal carotid artery and the M1 and M2 segments of the middle cerebral artery were divided into 3 groups: patients who received mechanical thrombectomy immediately (MT-I) or mechanical thrombectomy after early neurologic deterioration (MT-END) or intravenous thrombolysis only (IV). Due to the small sample size and the explorative and descriptive nature of the data, no statistical analysis was performed and data were presented either as frequencies, mean $\pm \mathrm{SD}$ for continuous data, or median with range for ordinal data.

Of 2213 patients in our recanalization data base with ischemic strokes in the anterior circulation, we identified 378 (17.1\%) with MSS (Fig 1). Of those, 250 (66\%) had an excellent outcome (mRS $0-1)$ and sICH occurred in 9 (2.4\%). One-hundred eighty-one of the patients with MSS (47.9\%) underwent either CT or MR angiography. Of those, 54 (29.8\%) patients had a large-vessel occlusion (ICA, M1, or M2) and 14 (7.7\%) had an occlusion of the distal ICA or combined ICA/M1 occlusions and 18 (9.9\%) had an M1 occlusion. Eight of $54(14.8 \%)$ were initially treated with thrombectomy (MT-I); 6/54 (11.1\%), after deterioration (MTEND); and 40/54 (74\%), with thrombolysis only (IV). Of these IV patients, another 3 experienced END and no thrombectomy was performed. Clinical baseline characteristics were similar in all groups. The On-line Table shows all relevant parameters. Neither onset-to-earliest treatment nor door-to-needle time was considerably different among the groups, but mean door-to-vessel time was much shorter in MT-I compared with MT-END (207 \pm 144 versus $663 \pm 534$ minutes) for obvious reasons. The median NIHSS was lower in MT-I at baseline and before thrombectomy (2 versus 4 and 4 versus 14). All (22/57) M2 occlusions were in the IV group. Three patients in the MT-I group did not receive thrombolysis, whereas all patients in the MT-END group were initially treated with thrombolysis. Acute carotid artery stent placement was performed in 50\% of patients in each MT group. Rates of successful recanalization were very similar between the MT-I and the MT-END groups (75\% versus 100\%). In only $22.5 \%(9 / 40)$ of patients receiving IV thrombolysis was follow-up MR vessel imaging performed. Of those, only 44.4 (4/9) showed signs of recanalization on MRA. MRA, however, is not comparable with a cerebral angiography.

No changes regarding the site and extent of occlusion were seen between the initial imaging and the endovascular treatment. Rates of excellent outcome (modified Rankin Scale 0-1) were highest in patients with MT-I (75\%) compared with the other groups (MT-END, 33.3\%; IV, 55\%). Excluding the M2 occlusions, thrombolysis accounted for an excellent functional outcome of merely $39 \%$, similar to MT-END with $33 \%$, but again in contrast to MT-I with $75 \%$. No sICH occurred in any group. In total, only 2 treated patients with MSS died within 3 months.

\section{DISCUSSION}

Patients presenting with MSS usually have a high chance of excellent outcome. ${ }^{8}$ However, patients with MSS and largevessel occlusions have a less favorable outcome than patients presenting with MSS without these major vessel occlusions. ${ }^{8,9}$ In our case series, we found that patients with MSS and largevessel occlusions had, if immediately treated with MT, slightly better outcomes compared with patients with MSS who were only thrombolyzed (75\% [MT-I] versus $66 \%$ for all our patients with MSS or 55\% for those with MSS with large-vessel occlusions). Delaying mechanical thrombectomy until neurologic deterioration seemed to lower the beneficial effect, with a rate of excellent outcome of only $33.3 \%$. The functional outcome of patients in the MT-I group was also very similar to that described in a previous report of patients with MSS presenting without large vessel occlusion who had been treated with IV only. ${ }^{9}$ In contrast, a meta-analysis of the 5 seminal thrombectomy studies did not show a significant effect in patients with NIHSS $\leq 10 .{ }^{1}$ However, few patients with NIHSS $\leq 10$ were analyzed. Considering that there was a high fraction $(55 \%$, $22 / 40$ ) of M2 occlusions in the IV group whereas all occlusions in the MT groups were located in the ICA or M1, the efficacy of thrombectomy may be even higher. According to our data, the rates of excellent outcomes were then the following: 75\% (MTI), 33\% (MT-END), and 39\% (IV). Clearly, in this series, the more recent use of (immediate) thrombectomy compared with thrombolysis alone was dependent on the increased availability and performance of thrombectomy since the introduction of modern stent retrievers (all our patients undergoing MT 
were treated with these) and our clinical experience with time. Also, the overall standard of care might have improved during that time.

The median NIHSS score at baseline, and expectedly before thrombectomy, in the MT-END group was considerably higher than in the MT-I group; this might have contributed to the reduced chance of an excellent outcome. Because there was no thrombus dislodgement observed between the initial imaging and the endovascular treatment, we presume that hemodynamic disturbances over time caused END. ${ }^{10}$ Fifty percent of all MT patients required carotid artery stenting, which is an interesting finding, also indicating that patients with MSS with combined ICA/MCA occlusions might benefit from immediate treatment. MT led to a similar recanalization rate in both groups; most important, no sICH occurred in either group, which is in line with the published data on recanalization and safety of the recent thrombectomy trials. ${ }^{2-6}$ Another interesting finding of this analysis is that of 181 patients with MSS who received acute vessel imaging, large vessel occlusion (ICA, M1, and M2) was detected in almost 30\%, indicating that this is not a rare finding.

Due to the small sample size, the retrospective and exploratory nature of our data must be interpreted with caution. Owing to these circumstances, no statistical analysis was performed and the data should be regarded as hypothesis-generating and descriptive. Our results do not support previous findings that suggested that patients with MSS due to large vessel occlusion being treated with MT have an increased rate of sICH and that only thrombolysis and not MT was associated with significantly greater chances of full recovery. ${ }^{11}$ In our opinion, patients with large vessel occlusion and MSS do represent a subgroup of patients in whom considerable uncertainty remains with regard to the optimal acute recanalization therapy (ie, thrombolysis only or MT only after deterioration or MT immediately). Our data indicate that further analysis of larger registries or maybe even randomized trials in these patients should be performed. Our data also suggest that every patient with acute ischemic stroke should receive immediate vessel imaging because in this cohort, approximately $30 \%$ of patients with MMS in the anterior circulation did have largevessel occlusion (ICA, M1, and M2).

Disclosures: Markus M. Möhlenbruch—UNRELATED: Board Membership: Codman; Payment for Lectures Including Service on Speakers Bureaus: MicroVention, Phenox, Stryker Neurovascular. Johannes Pfaff-UNRELATED: Payment for Lectures Including Service on Speakers Bureaus: Siemens Healthineers; Travel/Accommoda-
tions/Meeting Expenses Unrelated to Activities Listed: Stryker Neurovascular. Christian Herweh-UNRELATED: Payment for Lectures Including Service on Speakers Bureaus: Bristol-Myers Squibb; Travel/Accommodations/Meeting Expenses Unrelated to Activities Listed: travel and registration fees paid by Stryker Neurovascular. Peter A. Ringleb_UNRELATED: Consultancy: Advisory Board for Boehringer Ingelheim, Covidien, Daichii Sankyo; Payment for Lectures Including Service on Speakers Bureaus: lecture fees from Bayer, Boehringer Ingelheim, Pfizer, and Daiichi Sankyo. Simon Nagel_UNRELATED: Consultancy: Brainomix; Grants/Grants Pending: Brainomix*; Payment for Lectures Including Service on Speakers Bureaus: Bayer Healthcare, Pfizer, Medtronic; Travel/Accommodations/Meeting Expenses Unrelated to Activities Listed: Brainomix, Böhringer Ingelheim, Bayer Healthcare, Medtronic. *Money paid to the institution.

\section{REFERENCES}

1. Goyal M, Menon BK, van Zwam WH, et al; HERMES collaborators. Endovascular thrombectomy after large-vessel ischaemic stroke: a meta-analysis of individual patient data from five randomised trials. Lancet 2016;387:1723-31 CrossRef Medline

2. Goyal M, Demchuk AM, Menon BK, et al; ESCAPE Trial Investigators. Randomized assessment of rapid endovascular treatment of ischemic stroke. N Engl J Med 2015;372:1019-30 CrossRef Medline

3. Jovin TG, Chamorro A, Cobo E, et al; REVASCAT Trial Investigators. Thrombectomy within $\mathbf{8}$ hours after symptom onset in ischemic stroke. N Engl J Med 2015;372:2296-306 CrossRef Medline

4. Saver JL, Goyal M, Bonafe A, et al; SWIFT PRIME Investigators. Stent-retriever thrombectomy after intravenous t-PA vs. t-PA alone in stroke. $N$ Engl J Med 2015;372:2285-95 CrossRef Medline

5. Berkhemer OA, Fransen PS, Beumer D, et al. A randomized trial of intraarterial treatment for acute ischemic stroke. N Engl J Med 2015; 372:11-20 CrossRef Medline

6. Campbell BCV, Mitchell PJ, Kleinig TJ, et al; EXTEND-IA Investigators. Endovascular therapy for ischemic stroke with perfusion-imaging selection. $N$ Engl J Med 2015;372:1009-18 CrossRef Medline

7. Hacke W, Kaste M, Fieschi C, et al; Second European-Australasian Acute Stroke Study Investigators. Randomised double-blind placebo-controlled trial of thrombolytic therapy with intravenous alteplase in acute ischaemic stroke (ECASS II). Lancet 1998;352:1245-51 CrossRef Medline

8. Nedeltchev K, Schwegler B, Haefeli T, et al. Outcome of stroke with mild or rapidly improving symptoms. Stroke 2007;38: 2531-35 CrossRef Medline

9. Zhu W, Churilov L, Campbell BCV, et al. Does large vessel occlusion affect clinical outcome in stroke with mild neurologic deficits after intravenous thrombolysis? J Stroke Cerebrovasc Dis 2014;23:2888-93 CrossRef Medline

10. Tisserand M, Seners P, Turc G, et al. Mechanisms of unexplained neurological deterioration after intravenous thrombolysis. Stroke 2014;45:3527-34 CrossRef Medline

11. Urra X, San Román L, Gil F, et al; Catalan Stroke Code and Reperfusion Consortium (Cat-SCR). Medical and endovascular treatment of patients with large-vessel occlusion presenting with mild symptoms: an observational multicenter study. Cerebrovasc Dis 2014;38:418-24 CrossRef Medline 